\title{
Complete Methodology for Electrical Modeling of RFIC Packages
}

\author{
Tzyy-Sheng Horng, Member, IEEE, Sung-Mao Wu, and Charlie Shih
}

\begin{abstract}
A complete methodology based on broadband $S$-parameter measurement is proposed to establish the electrical models for radio-frequency integrated circuit (RFIC) packages. The research is focused on calibration of the test-fixture parasitics to obtain the intrinsic $S$-parameters from which an equivalent coupled lumped model can be extracted for any pair of package leads under test. Then a step-by-step optimization scheme is employed to construct an equivalent circuit for the whole package. A real example on modeling a 16-lead Thin Shrink Small Outline Package (TSSOP) has been demonstrated. The established model can account for various package effects at radio frequencies.
\end{abstract}

Index Terms-Think Shrink Small Outline Packages, RFIC packages, $S$-parameter measurement, electrical model.

\section{INTRODUCTION}

$\mathbf{I}$ $\mathrm{N}$ modern communication systems, there is a continuous trend toward higher microwave frequencies for RFICs to meet the ever increasing bandwidth for faster data transmission. At such high frequencies, the electrical parasitics for most packages become so evident that they degrade the performance of RFICs significantly. There is, therefore, a great need to establish an equivalent model that can account for all kinds of high-frequency effects, such as resonance, coupling, dispersion, and frequency-dependent losses. In the past, electrical modeling techniques for the packages based on simulation were used to construct the equivalent LCR matrices [1]-[4]. However, these matrices were mainly low-frequency results and possessed difficulties in interpreting all the above-mentioned phenomena effectively. Considering the package as a multiport microwave network, some literature [5]-[7] have demonstrated extraction of the equivalent circuits from measured $S$-parameters. Owing to the limited port numbers of the vector network analyzers, the measured models were always restricted to some critical leads. For modeling the package as a whole, more advanced $S$-parameter related techniques have been exploited in our previous works [8], [9].

This paper presents a complete methodology to model small size and low pin-count packages used for housing RFICs. The

\footnotetext{
Manuscript received September 9, 1999; revised August 24, 2001. This work was presented in part at the 49th Electronic Components and Technology Conference, San Diego, CA, 1999. This work was supported by Orient Semiconductor Engineering, Inc., Kaohsiung, Taiwan, R.O.C. and the National Science Council, Taiwan R.O.C., under Grant NSC87-2215-E-110-016.

T.-S. Horng and S.-M. Wu are with the Department of Electrical Engineering, National Sun Yat-Sen University, Taiwan, R.O.C. (e-mail: jason@ee.nsysu.edu.tw).

C. Shih is with the Design Technology Development Division, Silcon Integrated Systems Corporation, Hsinchu 300, Taiwan, R.O.C. (e-mail: charlies@sis.com.tw).

Publisher Item Identifier S 1521-3323(01)09960-9.
}

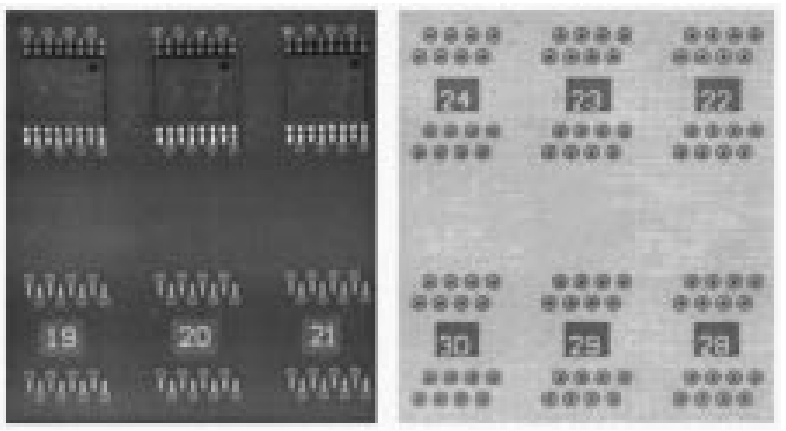

Fig. 1. Test-fixture for $S$-parameter measurment of the 16-lead TSSOP.

procedure begins with design and characterization of the test fixture made by printed circuit board (PCB) on which the packages are surface-mounted for measurement. The main function of the fixture is to provide good electrical and mechanical connection to the packages, and also to provide a good landing area for the microwave probes. In comparison with the small RFIC packages such as TSSOPs, the electrical length of the fixture is not negligible even though its PCB layout has been minimized in the fixture design. The fixture parasitics due to the multiple coupled striplines and via-holes can be analyzed using available simulation tools and verified by measurements. The cascaded microwave-network theory is then employed to remove the effects of the parasitics on the measured $S$-parameters. Our goal is to acquire the accurate $S$-parameters for any pair of coupled leads in the package. In the modeling each pair of coupled leads is represented by an equivalent coupled $\pi$ model. All the element quantities in the equivalent circuit can be extracted approximately from $S$-parameters at low frequencies and then optimized to best match the measured results over the entire frequency. Finally, another optimization is performed to combine individual coupled-lead models into one integrated model.

\section{S-PARAmETER MEASUREMENT}

Fig. 1 shows the test-fixture design for a 16-lead TSSOP. The package is soldered to one side of the fixture, while measurements are carried out on the other side by probing the via-holes. We employ Agilent's microwave design system (MDS) to characterize the fixture parasitics. As illustrated in Fig. 2, we can simulate the $S$-parameters for any pair of coupled striplines in the fixture in three different loaded configurations, which are denoted by open-path, short-path and loop-thru respectively. As an example, Fig. 3 shows comparison of the simulated results with measurements for the configuration shown in Fig. 2. Excellent agreement has been found up to $12 \mathrm{GHz}$. 


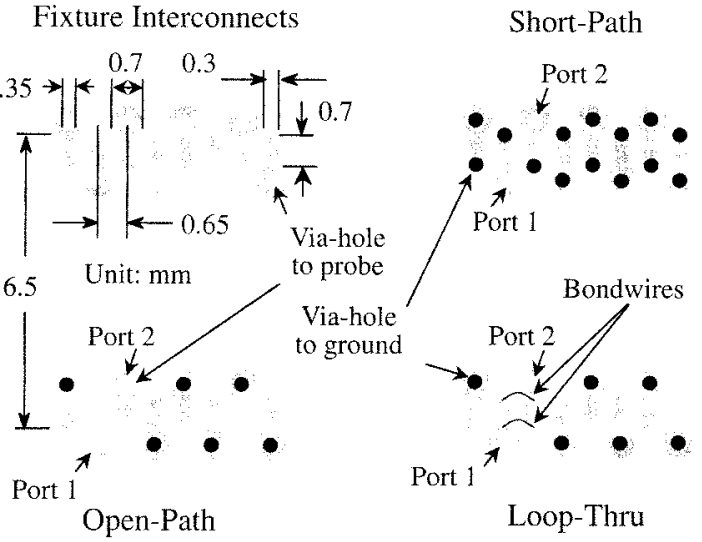

Fig. 2. Test-fixture interconnects in different loaded configurations.

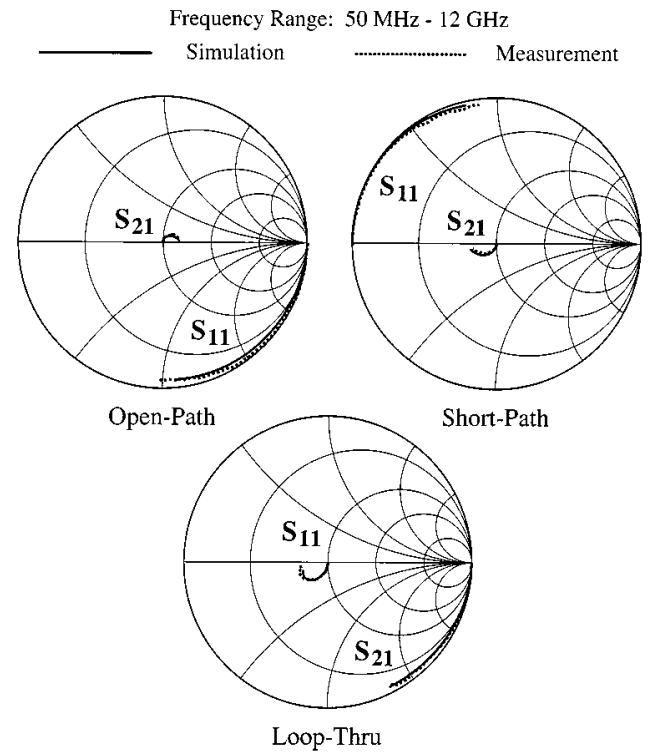

Fig. 3. Comparison of the simulated and measured $S$-parameters in electrical characterization of the test-fixture interconnects.

We measure the $S$-parameters again when the packages are surface-mounted to the test fixture. In a similar fashion, we consider loading the package leads in a variety of wire-bonding configurations. For example, in Fig. 4 the coupled leads 2 and 3 to be measured in the 16-lead TSSOP are left open-ended for the open-path configuration while both leads are wire-bonded to the die pad for the short-path configuration. In the loop-thru configuration, they are wire-bonded to each other from the bondfingers to form a loop. Note that the other unused leads are all wire-bonded to the die pad. This allows our measurement to be less susceptible to the external noises in the frequency range up to $12 \mathrm{GHz}$. Owing to the imperfect ground effects, some package leads tend to act like antennas and start radiating above $12 \mathrm{GHz}$. Fig. 5 depicts the calibration procedure for removing the fixture parasitics. From the theory of the cascaded microwave network, the ABCD matrix for combining package leads and fixture striplines can be expressed as

$$
\left[\begin{array}{ll}
A & B \\
C & D
\end{array}\right]_{P T}=\left[\begin{array}{ll}
A & B \\
C & D
\end{array}\right]_{L}\left[\begin{array}{cc}
A & B \\
C & D
\end{array}\right]_{P}\left[\begin{array}{cc}
A & B \\
C & D
\end{array}\right]_{R} .
$$

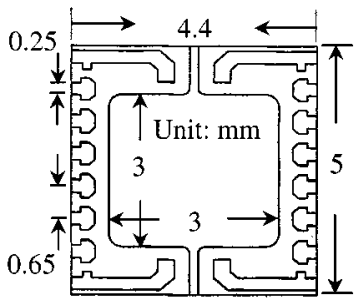

Outline

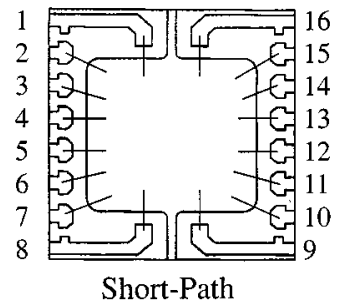

Short-Path

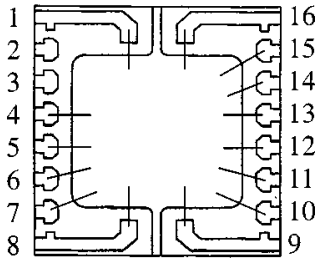

Open-Path

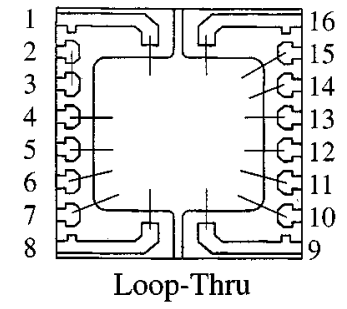

Fig. 4. Package coupled leads in different loaded configurations. The overall package length is $6.4 \mathrm{~mm}$ after including the outer lead portion.

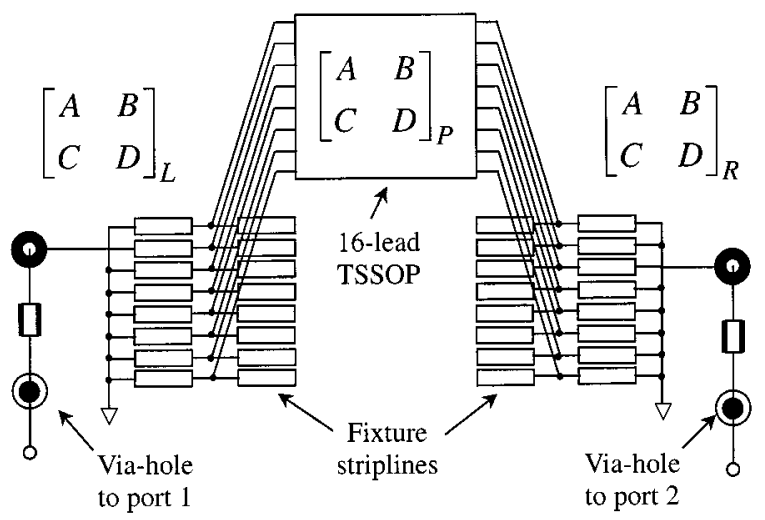

Fig. 5. Representation of the test-fixture interconnects and package coupled leads using cascaded microwave network.

On the right hand side of (1) the matrix with subscript $L$ represents the input fixture parasitics network, while the matrix with subscript $R$ represents the output fixture parasitics network. The matrix with subscript $P$ represents the intrinsic network associated with the package coupled leads. It is noted that such a cascaded network representation is under an assumption of negligible coupling among three networks. Since the models provided by MDS for the PCB via-holes and multiple coupled striplines have been verified by measurements, they can be used to generate the $\mathrm{ABCD}$ matrices of the input and output fixture parasitics networks. Consequently, the ABCD matrix for the coupled leads alone is found by

$$
\left[\begin{array}{ll}
A & B \\
C & D
\end{array}\right]_{P}=\left[\begin{array}{cc}
A & B \\
C & D
\end{array}\right]_{L}^{-1}\left[\begin{array}{cc}
A & B \\
C & D
\end{array}\right]_{P T}\left[\begin{array}{cc}
A & B \\
C & D
\end{array}\right]_{R}^{-1} .
$$

After transformation, the calibrated $S$-parameters for the coupled leads 2 and 3 are shown in Fig. 6. When compared to the $S$-parameters with effects of fixture parasitics, it is reasonable to see that the phase delays decrease obviously for the reflection coefficients in the open-path and short-path configurations, and also for the transmission coefficient in the loop-thru configuration. It is noted that the test fixture behaves more like 


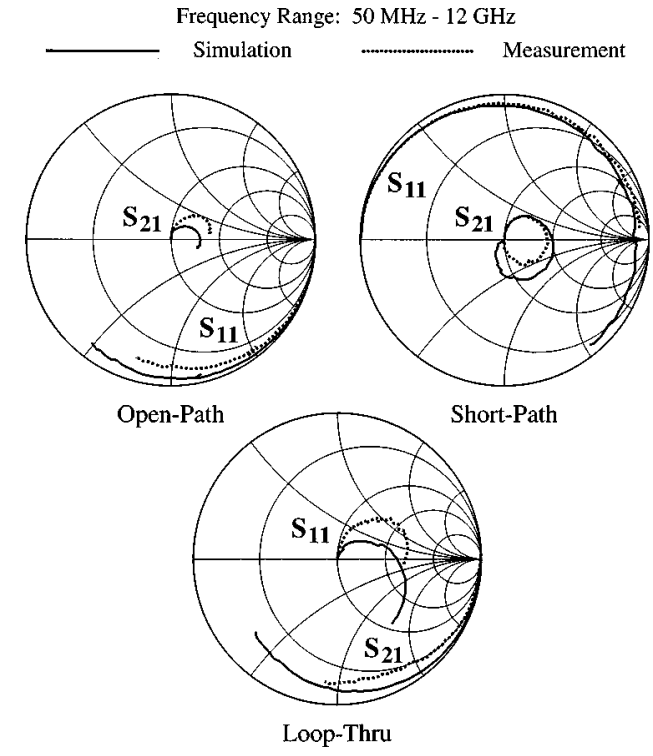

Fig. 6. Comparison of the $S$-parameters for package coupled leads before and after calibration.

the shunt capacitances connected at the input and output ports, which can lower the equivalent lead impedance to achieve better impedance match. This explains why the transmission loss in the loop-thru configuration becomes larger in the absence of the test fixture.

\section{EQUivalENT-CiRCUIT MODELING}

In consideration of simplicity and bandwidth, the coupled $\pi$ model was empirically found to be a good choice for characterization of the coupled leads in the package. As shown in Fig. 7, the coupled leads to be modeled are under four loaded configurations, i.e., open-path (op), short-path (sp), loop-thru (lt), and loop-ground (lg), for providing sufficient $S$-parameter information in extracting the equivalent-circuit elements. In the open-path configuration, it is found that the lead and mutual capacitances, $C_{i}, C_{j}, C_{i j}$, are the dominant factors to determine the $S$-parameters at low frequencies in the relations

$$
\begin{aligned}
S_{11,22}^{\text {op }} & \approx \frac{\left[j 2 \pi f\left(C_{i, j}+C_{i j}\right)\right]^{-1}-Z_{0}}{\left[j 2 \pi f\left(C_{i, j}+C_{i j}\right)\right]^{-1}+Z_{0}} \\
S_{21}^{\text {op }} & =S_{12}^{\text {op }} \approx \frac{2}{2+\left(j 2 \pi f C_{i j} Z_{0}\right)^{-1}}
\end{aligned}
$$

where $Z_{0}=50 \Omega$ is the impedance of the measurement system. From (3) and (4) the capacitances can be further approximated as

$$
\begin{aligned}
& C_{i, j}=\frac{-\tan \left(\angle S_{11,22}^{\mathrm{op}} / 2\right)}{2 \pi f Z_{0}}-C_{i j} \\
& C_{i j} \approx \frac{1}{4 \pi f Z_{0}} \frac{\left|S_{21}^{\mathrm{op}}\right|}{\sqrt{1-\left|S_{21}^{\mathrm{op}}\right|^{2}}} .
\end{aligned}
$$
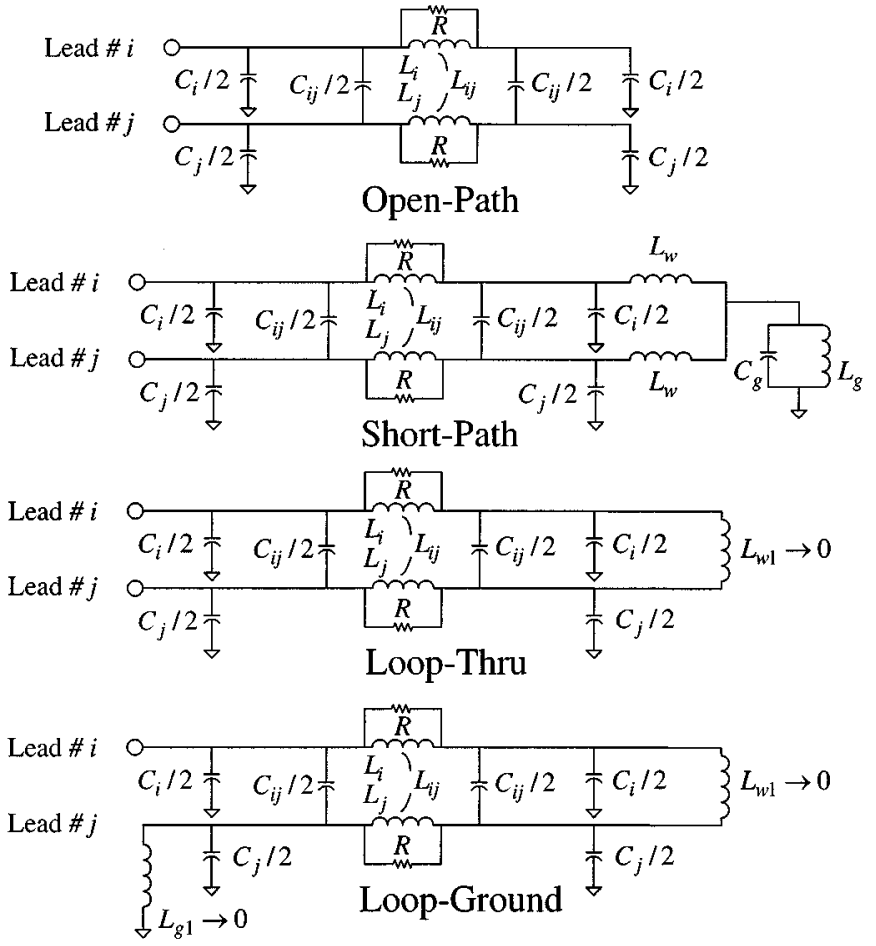

Fig. 7. Equivalent circuits for package coupled leads in different loaded configurations.

In the short-path configuration, the lead and mutual inductances $L_{i}, L_{j}, L_{i j}$, and bondwire inductance $L_{w}$, and the ground inductance $L_{g}$, dominate the $S$-parameter responses at low frequencies. Their relations are

$$
\begin{aligned}
S_{11,22}^{\mathrm{sp}} & \approx \frac{j 2 \pi f\left(L_{i, j}+L_{w}+L_{g}\right)-Z_{0}}{j 2 \pi f\left(L_{i, j}+L_{w}+L_{g}\right)+Z_{0}} \\
S_{21}^{\mathrm{sp}} & =S_{12}^{\mathrm{sp}} \approx \frac{j 4 \pi f\left(L_{i j}+L_{g}\right) Z_{0}}{Z_{0}^{2}+4 \pi^{2} f^{2}\left(L_{i j}+L g\right)^{2}} \approx \frac{j 4 \pi f\left(L_{i j}+L_{g}\right)}{Z_{0}} .
\end{aligned}
$$

From (7) and (8) we can solve for the lead and mutual inductances

$$
\begin{aligned}
L_{i, j} & \approx \frac{Z_{0}}{2 \pi f} \cot \left(\angle S_{11,22}^{\mathrm{sp}} / 2\right)-L_{w}-L_{g} \\
L_{i j} & \approx \frac{Z_{0}\left|S_{21}^{\mathrm{sp}}\right|}{4 \pi f}-L_{g} .
\end{aligned}
$$

For the loop-ground configuration, a loop inductance equivalent to $L_{i}+L_{j}-2 L_{i j}$ becomes the major contribution to the reflection coefficient at low frequencies. Thus,

$$
S_{11}^{\lg } \approx \frac{j 2 \pi f\left(L_{i}+L_{j}-2 L_{i j}\right)-Z_{0}}{j 2 \pi f\left(L_{i}+L_{j}-2 L_{i j}\right)+Z_{0}} .
$$

From (11) the loop inductance can be found as

$$
L_{i}+L_{j}-2 L_{i j} \approx \frac{Z_{0}}{2 \pi f} \cot \left(\angle S_{11}^{\mathrm{lg}} / 2\right)
$$




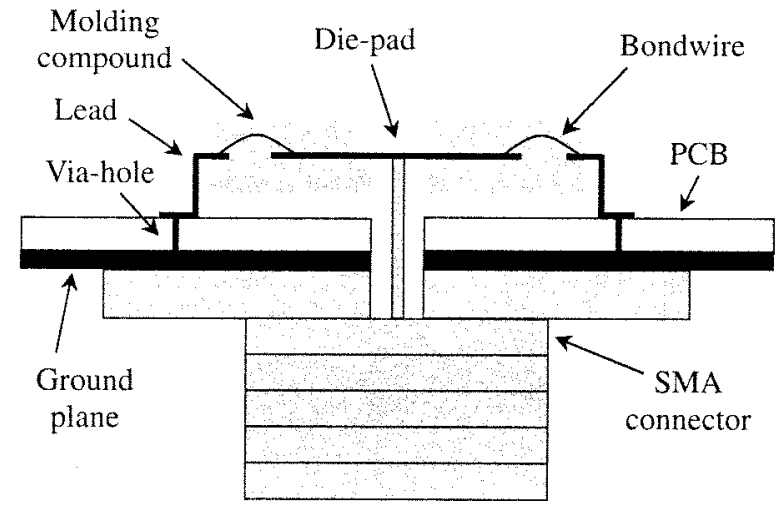

Fig. 8. Measurement of ground parasitics for TSSOPs.

TABLE I

EXTRACTED QUANTITIES OF EQUIVALENT-CIRCUIT ELEMENTS FOR THE COUPLED-LEAD MODEL

\begin{tabular}{c|c|c|c|c}
\hline$L_{2}$ & $L_{3}$ & $L_{23}$ & $L_{g}$ & $L_{w}$ \\
\hline $1.266 \mathrm{nH}$ & $1.127 \mathrm{nH}$ & $0.360 \mathrm{nH}$ & $0.150 \mathrm{nH}$ & $0.364 \mathrm{nH}$ \\
\hline$C_{2}$ & $C_{3}$ & $C_{23}$ & $C_{g}$ & $R$ \\
\hline $0.168 \mathrm{pF}$ & $0.109 \mathrm{pF}$ & $0.056 \mathrm{pF}$ & $0.92 \mathrm{pF}$ & $100 \mathrm{kOH}$ \\
\hline
\end{tabular}

Substitute (9) and (10) into (12), we can determine the bondwire inductance in the form

$$
\begin{aligned}
L_{w} \approx \frac{Z_{0}}{4 \pi f}\left[\cot \left(\angle S_{11}^{\mathrm{sp}} / 2\right)+\cot \left(\angle S_{22}^{\mathrm{sp}} / 2\right)\right. \\
\left.-\cot \left(\angle S_{11}^{\mathrm{gg}} / 2\right)-\left|S_{21}^{\mathrm{sp}}\right|\right] .
\end{aligned}
$$

The die pad in a TSSOP acts as a grounding device for RFICs. Its parasitics can be characterized using a simple parallel LC circuit with resonant frequency $f_{r}$ [8]-[10]. Fig. 8 shows the one-port $S$-parameter measurement for extraction of such ground parasitics. The packages under test are implemented by piercing the test fixture and molding compound with the center pin of a SMA connector. The pin tip and ground base of the SMA connector are soldered to the die pad and fixture's ground plane respectively. The ground inductance can be extracted from the measured phase of reflection coefficient $\left(\angle S_{11}^{g}\right)$ at low frequencies, which can be expressed as

$$
L_{g} \approx \frac{Z_{0}}{2 \pi f} \cot \left(\angle S_{11}^{g} / 2\right)
$$

With additional knowledge of the resonant frequency estimated from the measured reflection coefficient, the ground capacitance is determined as

$$
C_{g} \approx \frac{1}{4 \pi^{2} f_{r}^{2} L_{g}} .
$$

As an example, the extracted quantities of the equivalent-circuit elements using the above expressions for the coupled leads 2 and 3 in the 16-lead TSSOP are listed in Table I. The generated $S$-parameters from the equivalent circuits are further compared with the original measurement data, as shown in Fig. 9.

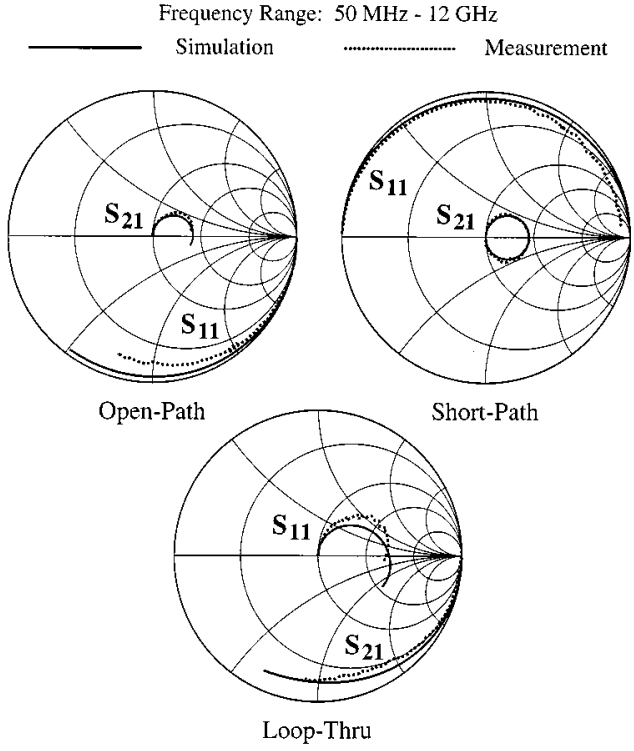

Fig. 9. Comparison of the simulated $S$-parameters based on the extracted coupled $\pi$ model with measurements for package coupled leads.

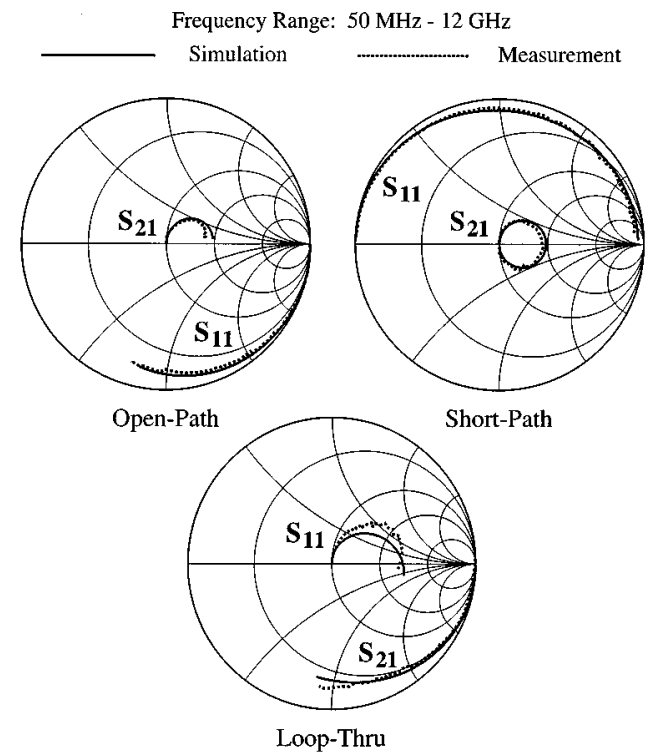

Fig. 10. Comparison of the simulated $S$-parameters based on the optimized coupled $\pi$ model with measurements for package coupled leads.

As expected, good agreement has been found at lower frequencies. However, considerable discrepancy has been also found at higher frequencies. Adopting these extracted quantities as an initial guess, a gradient optimizer in MDS has been used to find the optimum quantities by minimizing the sum of the absolute values of the difference between two sets of $S$-parameters. The optimization is performed up to $12 \mathrm{GHz}$ and the results for the $S$-parameters and optimum element quantities are shown in Fig. 10 and Table II respectively. One can compare to see that after optimization the quantities of lead inductance and capacitance decrease, while the quantities of mutual inductance and capacitance increase. The variation of these quantities implies that both inductive and capacitive coupling coefficients 
TABLE II

OPTIMIZED QUANTITIES OF EQUIVALENT-CIRCUIT ELEMENTS FOR THE COUPLED-LEAD MODEL

\begin{tabular}{c|c|c|c|c}
\hline$L_{2}$ & $L_{3}$ & $L_{23}$ & $L_{g}$ & $L_{w}$ \\
\hline $1.191 \mathrm{nH}$ & $1.045 \mathrm{nH}$ & $0.394 \mathrm{nH}$ & $0.150 \mathrm{nH}$ & $0.364 \mathrm{nH}$ \\
\hline$C_{2}$ & $C_{3}$ & $C_{23}$ & $C_{g}$ & $R$ \\
\hline $0.139 \mathrm{pF}$ & $0.094 \mathrm{pF}$ & $0.061 \mathrm{pF}$ & $0.92 \mathrm{pF}$ & $0.992 \mathrm{kOH}$ \\
\hline
\end{tabular}

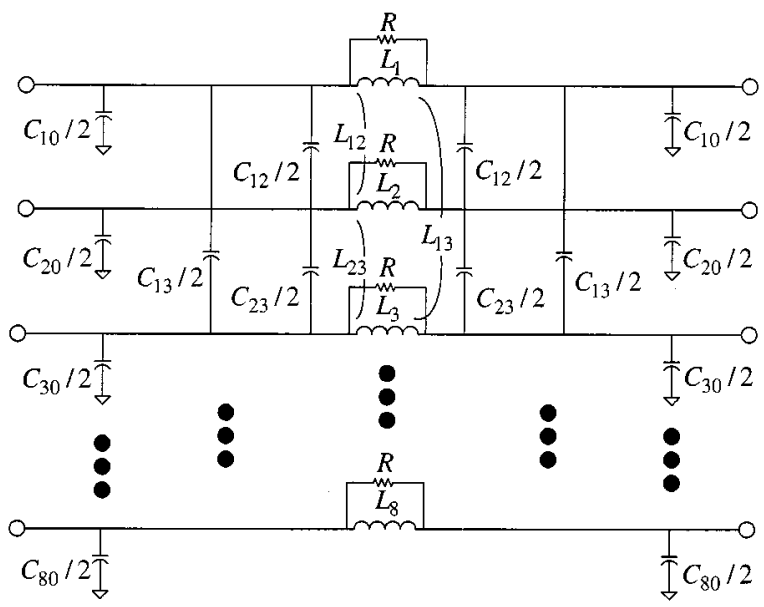

Fig. 11. Complete electrical model for the 16-lead TSSOP.

TABLE III

QUANTITIES OF EQUIVALENT-CIRCUIT ELEMENTS FOR COMPLETE ELECTRICAL MODEL OF THE 16-LEAD TSSOP

\begin{tabular}{c|c|c|c|c|c|c|c|c|c|c}
\hline $\begin{array}{c}C_{i j}(\mathrm{pF}) \\
L_{i j}(\mathrm{nH})\end{array}$ & $j=1$ & $j=2$ & $j=3$ & $j=4$ & $j=5$ & $j=6$ & $j=7$ & $j=8$ & $j=0$ & $C_{i L}(\mathrm{pF})$ \\
\hline$i=1$ & & 0.065 & 0.006 & 0.001 & 0 & 0 & 0 & 0 & 0.168 & 0.240 \\
\hline$i=2$ & 0.436 & & 0.061 & 0.005 & 0.001 & 0 & 0 & 0 & 0.054 & 0.186 \\
\hline$i=3$ & 0.209 & 0.394 & & 0.061 & 0.005 & 0.001 & 0 & 0 & 0.036 & 0.170 \\
\hline$i=4$ & 0.114 & 0.187 & 0.394 & & 0.061 & 0.005 & 0.001 & 0 & 0.036 & 0.170 \\
\hline$i=5$ & 0.067 & 0.103 & 0.187 & 0.394 & & 0.061 & 0.005 & 0.001 & 0.036 & 0.170 \\
\hline$i=6$ & 0.039 & 0.061 & 0.103 & 0.187 & 0.394 & & 0.061 & 0.006 & 0.036 & 0.170 \\
\hline$i=7$ & 0.019 & 0.035 & 0.061 & 0.103 & 0.187 & 0.394 & & 0.065 & 0.054 & 0.186 \\
\hline$i=8$ & 0.009 & 0.019 & 0.039 & 0.067 & 0.114 & 0.209 & 0.436 & & 0.168 & 0.240 \\
\hline$L_{j}(\mathrm{nH})$ & 1.751 & 1.238 & 1.121 & 1.121 & 1.121 & 1.121 & 1.238 & 1.751 & &
\end{tabular}

tend to increase with frequency. To include the frequency-dependent losses resulting from the skin effects and dielectric loss, an optimum resistance $R$ in parallel with the inductance for the individual lead has been also simulated.

After repeating the measurement and modeling of a number of coupled leads, we have established a complete electrical model for the 16-lead TSSOP. Owing to symmetry, we consider the equivalent circuit for one half of the package, as shown in Fig. 11. Note that the quantities for lead inductance and capacitance depend on the loaded conditions of the adjacent leads and are slightly different from those found in modeling the coupled leads only. Again, we can use the optimizer to determine their optimum quantities. Finally, we finish the entire procedure for modeling the 16-lead TSSOP and demonstrate all the equivalent-circuit element quantities in Table III. A

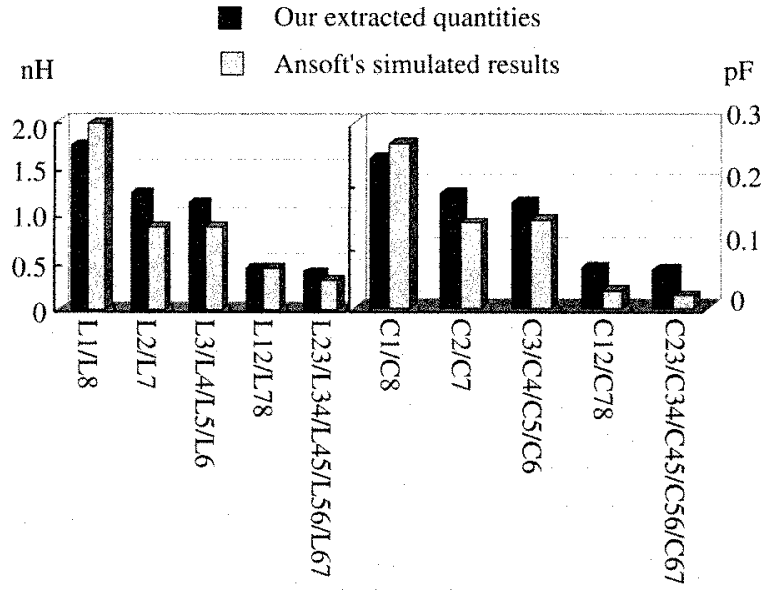

Fig. 12. Comparison of the extracted equivalent-circuit parameters based on our approach with the Ansoft simulations.

comparison with the quantities calculated using the Boundary Element Method (BEM) in Ansoft's Maxwell Q3D Extractor has been provided in Fig. 12. In general our extracted quantities agree well with Ansoft's simulated results except the mutual capacitances. It is noted that the Ansoft simulation is based on a quasistatic approach, which usually underestimates the capacitive coupling at high frequencies.

\section{CONCLUSION}

A complete and rigorous technique for electrical modeling of RFIC packages has been presented. The procedure includes the test-fixture design and characterization, coupled-lead $S$-parameter measurement and calibration, and optimization schemes for establishing the equivalent circuit of the whole package. An equivalent circuit for a 16-lead TSSOP has been developed as an example. The simulated $S$-parameters for many coupled leads loaded in different configurations have been matched against measurements. Good agreement can be found over a wide frequency range up to $12 \mathrm{GHz}$.

\section{REFERENCES}

[1] F. Ndagijimana, J. Engdahl, A. Ahmadouche, and J. Chilo, "Frequency limitation on an assembled SOP8 package," in Proc. 43rd Electron. Comp. Technol. Conf., 1993, pp. 530-535.

[2] A. C. Cangellaris, J. L. Prince, and L. P. Vakanas, "Frequency-dependent inductance and resistance calculation for three-dimensional structures in high-speed interconnect systems," IEEE Trans. Comp., Hybrids, Manufact. Technol., vol. 13, pp. 154-159, Mar. 1990.

[3] T. Y. Chou and Z. J. Cendes, "Capacitance calculation of IC packages using the finite element method and plane of symmetry," IEEE Trans. Computer-Aided Design, vol. 13, pp. 1159-1166, Sept. 1994.

[4] M. Caggiano, "A computer program that generates an RF electrical model of the parasitics of QFP packages," in Proc. 47th Electron. Comp. Technol. Conf., 1997, pp. 1146-1151.

[5] C. T. Tsai, "Package inductance measurement at high frequencies," in Proc. 42nd Electron. Comp. Technol. Conf., 1992, pp. 740-744.

[6] F. Mernyei, "Measurement and field simulation based characterization of plastic packages," Proc. IEEE 6th Topical Elect. Performance Electron. Packag. Meeting, pp. 181-184, 1997.

[7] A. Pham, C. Chun, J. Laskar, and B. Hutchison, "Surface mount microwave package characterization technique," in IEEE MTT-S Int. Microwave Symp. Dig., 1997, pp. 995-998.

[8] T. S. Horng, S. M. Wu, and C. Shih, "Electrical modeling of RFIC packages up to $12 \mathrm{GHz}$," in Proc. 49th Electron. Comp. Technol. Conf., 1999, pp. 867-712. 
[9] - "Advanced measurement techniques for generation of SPICE models of RFIC packages," Electron. Lett., vol. 35, pp. 1945-1946, Oct. 1999.

[10] T. S. Horng and S. M. Wu, "Accurate measurement of ground-ring inductance in ball grid array package," Electron. Lett., vol. 35, pp. 520-521, Sept. 1999.

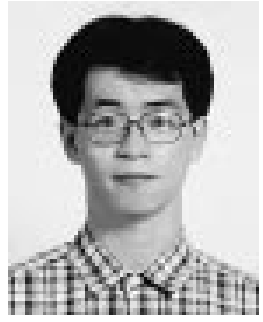

Tzyy-Sheng Horng (S'88-M'92) was born December 7, 1963, in Taichung, Taiwan, R.O.C. He received the B.S.E.E. degree from National Taiwan University, Taipei, in 1985, and the M.S.E.E. and Ph.D. degrees from the University of California, Los Angeles, in 1990 and 1992, respectively.

$\mathrm{He}$ is currently an Associate Professor in the Department of Electrical Engineering, National Sun Yat-Sen University, Kaohsiung, Taiwan. His research interests are in the area of RF and microwave integrated circuits and packages.

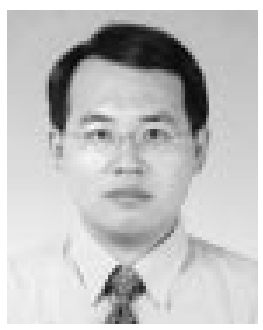

Sung-Mao Wu was born January 1, 1971, in Kaohsiung, Taiwan, R.O.C. He received the B.S.E.E degree from the Fu Jen Catholic University, Taipei, Taiwan, in 1994, the M.S.E.E. degree from the Yunlin University of Science and Technology, Yunlin, Taiwan, in 1996, and is currently pursuing the Ph.D. degree in electrical engineering at National Sun Yat-Sen University, Kaohsiung.

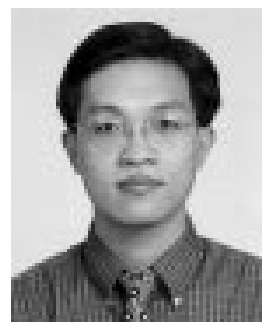

Charlie Shih was born May 5, 1970, in Hsin Chu, Taiwan, R.O.C. He received the B.S. degree from the Department of Electrophysics and the M.S. degree from the Institute of Electronics Engineering, National Chiao Tung University, Hsinchu, Taiwan, in 1992 and 1994, respectively.

He is currently a Mixed-Signal Circuit Design Engineer in the Design Technology Development Division, Silcon Integrated Systems Corp., Hsinchu. His research interests include passive interconnect modeling and RF circuits for wireless communications. 